Pacific Journal of Mathematics

ZEROS OF SUCCESSIVE ITERATES OF 


\title{
ZEROS OF SUCCESSIVE ITERATES OF MULTIPLIER-SEQUENCE OPERATORS
}

\author{
C. L. Prather and J. K. Shaw
}

\begin{abstract}
The authors study the zero free regions of iterates of multiplier-sequence operators for (i) functions analytic in the disc $|z|<R$ and (ii) functions analytic in $|z|>T$. Integral representations for the iterates of each class of functions are given. As a consequence the authors give a generalization of the Post-Widder inversion formula. Other applications include an investigation of the zero free regions for iterates of fractional integrals as well as connections between results obtained here and recent final set results for iterates of operators on balanced sums.
\end{abstract}

1. Introduction. Let $f(z)=\sum_{k=0}^{\infty} a_{k} z^{k}$ be analytic in a neighborhood of 0 and define an operator $\theta$, associated with a set of constants $\left\{\alpha_{k}\right\}_{k=0}^{\infty}$ and an integer $p \geq 0$, on the power basis by $\theta\left(z^{k}\right)=\alpha_{k} z^{k+p}$, $k=0,1,2, \ldots$ Thus for suitably restricted $\left\{\alpha_{k}\right\}$ we may write $(\theta f)(z)=$ $\sum_{k=0}^{\infty} \alpha_{k} a_{k} z^{k+p},\left(\theta^{2} f\right)(z)=\sum_{k=0}^{\infty} \alpha_{k} \alpha_{k+p} a_{k} z^{k+2 p}$, and so on. We call an operator of this type a multiplier-sequence operator. For example, if $\alpha_{k}=k$, then $(\theta f)(z)=z^{k+p} f^{\prime}(z)$; that is,

$$
\theta=z^{1+p} \frac{d}{d z} .
$$

In [13] (and in [14] for $f$ analytic in a neighborhood of $\infty$ ) the present authors studied the differential operators (1.1) and their zero-free regions, that is, regions $V_{n}$ in the plane such that $\left(\theta^{n} f\right)(z) \neq 0$ for $z \in V_{n}$ and all $n$ sufficiently large. Through change-of-variable methods, descriptions of $V_{n}$ are transmitted to the classical context of zero-free regions for ordinary derivatives. For instance, when $p=1$ and $F(z)=f(1 / z)$, then $f(z)=$ $F(1 / z),(\theta f)(z)=z^{2} f^{\prime}(z)=-F^{\prime}(1 / z)$, and $\left(\theta^{n} f\right)(z)=(-1)^{n} F^{(n)}(1 / z)$ in general. Therefore the regions $V_{n}^{-1}=\left\{z^{-1}: z \in V_{n}\right\}$ are free of zeros of $F^{(n)}$, for large enough $n$. The regions $V_{n}$ containing $z=0$ are discs which shrink with increasing $n$ to 0 . Their radii have orders of magnitude $V_{n}=1 / n$.

In the present paper we study zero-free regions for rather general multiplier-sequence operators. In particular, we realize all of the results of [13] and a portion of those of [14] as special cases. Additionally, we present a result on the successive iterates of fractional integrals, and we indicate a method which can be used for locating regions void of zeros of certain Special Functions (not their derivatives). Included among the applications and examples is a generalized Post-Widder transform inversion formula for multilplier-sequence operators. Finally, we connect our 
theorems to some results in the literature $([10],[11])$ on final sets for differential operators of a certain type.

2. Main results. We shall work with more general operators $\theta$ and functions $f(z)$ than those indicated above. Fix an integer $M$ (positive, negative or zero) and let $f(z)$ be given by

$$
f(z)=\sum_{k=-M}^{\infty} a_{k} z^{k}, \quad 0<|z|<R .
$$

If $M \leq 0, z=0$ is allowed. Let $p$ be a nonnegative number. Let $\alpha$ be a function which is defined on all numbers of the form $k+j p$, for $k=-M$, $-M+1,-M+2, \ldots$ and $j=0,1,2, \ldots$ If $p$ is an integer, $\alpha$ is of course a sequence indexed by the integers $k \geq-M$. We will denote the value of $\alpha$ at $k+j p$ by $\alpha_{k+j p}$ even though $p$ may not be integral. Let an operator $\theta$ be defined by its action on powers of $z$ by $\theta\left(z^{\nu}\right)=\alpha_{\nu} z^{\nu+p}$, where $\nu$ is of the form $k+j p$ as above. The domain of $\alpha$ is such that the successive iterates $\theta^{n}\left(z^{\nu}\right)=\alpha_{\nu} \alpha_{\nu+p} \cdots \alpha_{\nu+(n-1) p} z^{\nu+n p}$ are all defined for $n=1,2,3, \ldots$ Viewed as an analytic function $z^{\nu=n p}$ may be interpreted as the principal branch of the power function, although this will not be important in what follows.

Applying $\theta$ to (2.1), we have formally that $(\theta f)(z)=\sum_{k=-M}^{\infty} \alpha_{k} a_{k} z^{k+p}$, and introducing the notation

$$
\begin{aligned}
& A_{k, n}=\alpha_{k} \alpha_{k+p} \cdots \alpha_{k+(n-1) p}, \\
& \quad k=-M,-M+1, \ldots, n=1,2,3, \ldots,
\end{aligned}
$$

we may write, again formally,

$$
\left(\theta^{n} f\right)(z)=\sum_{k=-M}^{\infty} A_{k, n} a_{k} z^{k+n p}, \quad n=1,2,3, \ldots
$$

Note that the factors $A_{k, n}=A_{k, n}(p)$ depend on the number $p$, which we omit to simplify notation.

We now state our first hypotheses and make rigorous the formula (2.3). We will assume throughout this paper that

$$
\alpha_{-M} \neq 0 \text {, }
$$

and that

$$
\alpha_{k} \neq 0 \text { implies that } \alpha_{k+j p} \neq 0 \text { for } j=0,1,2, \ldots,
$$

where $k=-M,-M+1, \ldots$ We will assume that there is a first integer $P \geq 1$ such that

$$
\alpha_{-M+P} \neq 0
$$


Then it follows that

$$
A_{-M, n} \neq 0, a_{-M+P, n} \neq 0 \quad n=1,2,3, \ldots,
$$

but if $P>1$ then the definition of $A_{k, n}$ implies that

$$
A_{-M+1, n}=A_{-M+2, n}=\cdots=A_{-M+P-1, n}=0, \quad n=1,2,3, \ldots
$$

Our assumptions on the growth of the function $\alpha$ come in the form of two alternate hypotheses, the first of which we now state.

Hypothesis I. The set $\left\{\alpha_{\nu}\right\}$ satisfies $\alpha_{k+j p} \geq 0$ for all $k$ and $j$, and

$$
\begin{aligned}
& \frac{\alpha_{-M+P+j+n p}}{\alpha_{-M+n p}}\left[\frac{\alpha_{-M+n p}}{\alpha_{-M+P+n p}}\right]^{P+j} \leq 1, \\
& \quad j=0,1,2, \ldots ; n=0,1,2, \ldots .
\end{aligned}
$$

Under this condition, the factors $A_{k, n}$ satisfy

$$
\begin{aligned}
& \frac{A_{-M+P+j, n+1}}{A_{-M, n+1}}\left[\frac{A_{-M, n+1}}{A_{-M+P, n+1}}\right]^{P+j} \\
& \quad=\left[\frac{\alpha_{-M+P+j} \cdots \alpha_{-M+P+j+n p}}{\alpha_{-M} \cdots \alpha_{-M+n p}}\right]\left[\frac{\alpha_{-M} \cdots \alpha_{-M+n p}}{\alpha_{-M+P} \cdots \alpha_{-M+P+n p}}\right]^{P+j} \\
& \quad \leq 1, \text { for } n, j=0,1,2, \ldots
\end{aligned}
$$

Written another way, this becomes

$$
\frac{A_{-M+P+j, n}}{A_{-M, n}} \leq\left[\frac{A_{-M+P, n}}{A_{-M, n}}\right]^{P+j}, \quad n=1,2,3, \ldots ; j=0,1,2, \ldots
$$

Let $S_{-M+P+j, n+1}$ denote the term on the left of $(2.10)$.

LEMMA 2.1. For each fixed $j$, the sequence $\left\{S_{-M+P+j, n+1}\right\}_{n=0}^{\infty}$ is convergent.

Proof. First, we have $0 \leq S_{-M+P+j, n+1} \leq 1$ by (2.10). To obtain $S_{-M+P+j, n+1}$ from $S_{-M+P+j, n}$ we multiply it by the factor on the left of (2.9). By this inequality, we see that $\left\{S_{-M+P+j, n+1}\right\}$ is non-increasing for $n \rightarrow \infty$. This completes the proof.

We introduce now the notation

$$
R_{n}=\frac{A_{-M, n}}{A_{-M+P, n}}, \quad n=1,2,3, \ldots
$$


and the function

$$
H_{n}(w)=1+\sum_{j=0}^{\infty} S_{-M+P+j, n} w^{P+j}, \quad n=1,2,3, \ldots,|w|<1 .
$$

TheORem 2.1. Assume Hypothesis I and let $f(z)$ be given by (2.1). Then $\left(\theta^{n} f\right)(z)$, as given by (2.3), is defined for $0<|z|<\left(R_{n} / \rho\right)$ and is also given by

$$
\begin{array}{r}
\left(\theta^{n} f\right)(z)=(2 \pi i)^{-1} A_{-M, n} z^{-M+n p} \int_{|t|=\rho}(1 / t)^{M+1} f(1 / t) H_{n}\left(z t / R_{n}\right) d t \\
n=1,2,3, \ldots,
\end{array}
$$

where $(1 / \rho)<R$.

Proof. We define $\theta f, \theta^{2} f, \ldots$ by (2.3). What we have to show is that (2.3) converges for $0<|z|<\left(R_{n} / \rho\right)$ and that (2.12) holds there. By (2.7) and (2.8), the series in (2.3) has partial sums

$$
\begin{aligned}
\sum_{k=-M}^{m} A_{k, n} a_{k} z^{k+n p} & \\
& =A_{-M, n} a_{-M} z^{-M+n p}+\sum_{j=0}^{m+M-P} A_{-M+P+j, n} a_{-M+P+j} z^{-M+P+j+n p} \\
& =A_{-M, n} z^{-M+n p}\left\{a_{-M}+\sum_{j=0}^{m+M-P} \frac{A_{-M+P+j, n}}{A_{-M, n}} a_{-M+P+j} z^{P+j}\right\}
\end{aligned}
$$

In view of (2.11) we have

$$
\frac{A_{-M+P+j, n}}{A_{-M, n}}|z|^{P+j} \leq\left(|z| / R_{n}\right)^{P+j}
$$

and this implies convergence of (2.3) in $0<|z|<R_{n} R$. The convergence is uniform and absolute on compact sets.

Now define the function $K_{n}(w)=\sum_{k=-M}^{\infty} A_{k, n} w^{k+M}$; this is defined for $|w|<R_{n}$, according to (2.11). Writing $w=z t$,

$$
K_{n}(z t)=\sum_{k=-M}^{\infty} A_{k, n} z^{k+M} t^{k+M}, \quad|z t|<R_{n},
$$

and the Cauchy Integral Formula yields

$$
A_{k, n} z^{k+M}=(2 \pi i)^{-1} \int_{|t|=\rho} \frac{K_{n}(z t)}{t^{k+M+1}} d t .
$$


This is valid when $|z|<R_{n} / \rho$. If we require $(1 / \rho)<R$, then $|z|<R_{n} / \rho$ implies $|z|<R_{n} R$ also, and so (2.13) may be substituted into (2.3) when $0<|z|<R_{n} / \rho$. This gives

$$
\begin{aligned}
\left(\theta^{n} f\right)(z) & =z^{-M+n p} \sum_{k=-M}^{\infty} A_{k, n} a_{k} z^{k+M} \\
& =z^{-M+n p}(2 \pi i)^{-1} \sum_{k=-M}^{\infty} a_{k} \int_{|t|=\rho}\left(K_{n}(z t) / t^{k+M+1}\right) d t \\
& =z^{-M+n p}(2 \pi i)^{-1} \int_{|t|=\rho}(1 / t)^{M+1} f(1 / t) K_{n}(z t) d t
\end{aligned}
$$

where we have used uniform convergence of the series to integrate termwise. Note that $f(1 / t)$ is defined because $|1 / t|=(1 / \rho)<R$. In (2.14) put $z=R_{n} x$, note (2.7) and (2.8), and expand $K_{n}(z t)$ to produce

$$
\begin{aligned}
\left(\theta^{n} f\right)(z)= & A_{-M, n} z^{-M+n p}(2 \pi i)^{-1} \int_{|t|=\rho}(1 / t)^{M+1} f(1 / t) \\
& \times\left\{1+\sum_{j=0}^{\infty} \frac{A_{-M+P+j, n}}{A_{-M, n}}\left[\frac{A_{-M, n}}{A_{-M+P, n}} x t\right]^{P+j}\right\} d t
\end{aligned}
$$

which is equivalent to (2.12).

THEOREM 2.2 Assume Hypothesis I, let $f(z)$ be given by (2.1), and let $a_{-M} \neq 0$. Then there exists a constant $\gamma, 0<\gamma<(1 / \rho)<R$, such that for all $n$ sufficiently large $\left(\theta^{n} f\right)(z)$ has no zero in $0<|z| \leq R_{n} \gamma$.

Proof. Writing $z=R_{n} x$ in (2.12), we have

$$
\begin{aligned}
\frac{z^{M-n p}\left(\theta^{n} f\right)(z)}{A_{-M, n}} & =(2 \pi i)^{-1} \int_{|t|=\rho}(1 / t)^{M+1} f(1 / t) H_{n}(x t) d t \\
& =I_{n}(x),
\end{aligned}
$$

with $I_{n}(x)$ defined in the obvious way. This is valid for $0<|x t|=$ $R_{n}^{-1}|z t|<R_{n}^{-1}\left(R_{n} / \rho\right) \rho=1$, and consequently $I_{n}(x)$ is defined for $|x|<$ $(1 / \rho)$. By Lemma 2.1 and the definition of $H_{n}(w)$, the sequence $\left\{H_{n}(w)\right\}_{n=1}^{\infty}$ converges uniformly on compact subsets of $|w|<1$ to a function

$$
H(w)=1+H_{P} w^{P}+H_{P+1} w^{P+1}+\cdots=\sum_{m=0}^{\infty} H_{m} w^{m} .
$$


Holding $x$ to a compact subset of $|x|<(1 / \rho)$ and letting $n \rightarrow \infty$ in (2.15), we obtain a limit function for $\left\{I_{n}(x)\right\}_{n=1}^{\infty}$,

$$
\text { (2.16) } I(x)=(2 \pi i)^{-1} \int_{|t|=\rho}(1 / t)^{M+1} f(1 / t) H(x t) d t, \quad|x|<(1 / \rho)
$$

that is

$$
\text { (2.17) } \lim _{n \rightarrow \infty}\left\{\frac{\left(R_{n} x\right)^{M-n p}\left(\theta^{n} f\right)\left(R_{n} x\right)}{A_{-M, n}}\right\}=I(x), \quad|x|<(1 / \rho) \text {, }
$$

with uniform convergence on compact sets. An easy calculation gives

$$
I(x)=\sum_{m=0}^{\infty} H_{m} a_{-M+m} x^{m}=\sum_{m=0}^{\infty} I_{m} x^{m}
$$

and in particular $I_{0}=a_{-M} \neq 0$. Thus we can find a neighborhood $|x| \leq \gamma$ $<(1 / \rho)$ in which $I(x) \neq 0$. The conclusion of the theorem follows from (2.17).

If $M \leq 0$ the requirement $z \neq 0$ can be dropped from the theorem. Also the assumption $a_{-M} \neq 0$ can be weakened to $H_{m} a_{-M+m} \neq 0$ for some $m$; i.e., $I(x)$ does not vanish identically. For in this case we can find a neighborhood $|x| \leq \gamma$ in which $I(x)$ has no zero other than (possibly) $x=0$. Finally, we remark that the multiple-valued nature of the terms $z^{n p}$ for non-integral $p$ is immaterial in the theorem, for these can always be factored out as in (2.14).

From one point of view, (2.17) may be regarded as a transform inversion formula, in the sense of the classical Post-Widder formula ([9], [15], [16]). To illustrate, let us assume that $P=1$ and that $H_{m} \neq 0$ for all $m$, under Hypothesis I. Let a measure $\mu$ of bounded variation on $[0, \infty)$ solve the Stieltjes moment problem

$$
H_{k}^{-1}=\int_{0}^{\infty} \tau^{k} d \mu(\tau), \quad k=0,1,2, \ldots ;
$$

see [16, p. 139] for details. If $I(x)$ is an entire function, and $f(z)$ is given by $(2.1)$, then we have

$$
\begin{aligned}
z^{M} f(z) & =\sum_{k=-M}^{\infty} a_{k} z^{k+M}=\sum_{k=-M}^{\infty}\left(I_{k+M} / H_{k+M}\right) z^{k+m} \\
& =\sum_{k=-M}^{\infty} I_{k+M} \int_{0}^{\infty}(z \tau)^{k+M} d \mu(\tau) \\
& =\int_{0}^{\infty} I(z \tau) d \mu(\tau)
\end{aligned}
$$


provided the integral exists and termwise integration can be justified. We may interpret (2.18) as a transform equation: $\hat{f}(z)=z^{M} f(z)$ is the " $\theta$ transform" of $I(x)$. The reciprocal relation is given by (2.16), which can be written as

$$
I(x)=(2 \pi i)^{-1} \int_{|t|=\rho}(1 / t) \hat{f}(1 / t) H(x t) d t .
$$

Consider the example $M=-1, \alpha_{k}=k, p=1, P=1$. Hypothesis $\mathrm{I}$ is easily verified, and we calculate that

$$
S_{2+j, n}=\frac{(n+1)(n+2) \cdots(n+j+1)}{(n+1)^{j+1}(j+1) !} \rightarrow \frac{1}{(j+1) !}, \quad n \rightarrow \infty,
$$

and so $H_{k}^{-1}=k$ ! The corresponding moment problem is solved by $k$ ! = $\int_{0}^{\infty} \tau^{k} e^{-\tau} d \tau$. Here (2.18) is easily justified and becomes after a change of variable

$$
f(1 / z)=\int_{0}^{\infty} e^{-z t} I(t) d t
$$

implying that $F(z)=f(1 / z)$ is the Laplace transform of $I(t)$. From $\alpha_{k}=k$ and $p=1$ it follows that $\theta=z^{2} d / d z$. Now $\left(\theta^{n} f\right)(z)=$ $(-1)^{n} F^{(n)}(1 / z)$ as noted earlier. Since $R_{n}=(n+1)^{-1},(2.17)$ reduces in this case to

$$
\lim _{n \rightarrow \infty} \frac{(-1)^{n}}{n !}\left(\frac{n+1}{x}\right)^{n+1} F^{(n)}\left(\frac{n+1}{x}\right)=I(x) .
$$

This was first derived by E. L. Post in 1930 [9, p. 772]. See [15], [16], [17], [18] for further references.

3. Alternate hypothesis: If the terms $\left\{\alpha_{\nu}\right\}$ are negative or complex, the previous results do not apply. We will modify them by using an alternate set of growth conditions. We still assume (2.4), (2.5) and (2.6), so that (2.7) and (2.8) hold. The following replaces (2.9).

Hypothesis II. There exists a number $S>0$ such that

$$
\left|\frac{\alpha_{-M+P+j+n p}}{\alpha_{-M+n p}}\right|\left|\frac{\alpha_{-M+n p}}{\alpha_{-M+P+n p}}(1 / S)\right|^{P+j} \leq 1 ; \quad n, j=0,1,2, \ldots
$$

Instead of (2.11), but proving it in the same way, we now have

$$
\left|\frac{A_{-M+P+j, n}}{A_{-M, n}}\right| \leq\left(S^{n} / R_{n}\right)^{P+j}, \quad n=1,2,3, \ldots ; j=0,1,2, \ldots,
$$


where $R_{n}=\left|A_{-M, n} / A_{-M+P, n}\right|$ as before. This time $\left(\theta^{n} f\right)(z)$, still given by (2.3), is defined for $0<|z|<R_{n} R / S^{n}$. The function $K_{n}(w)$ will be defined as before except that $|w|<R_{n} / S^{n}$, and for (2.13) we must insist that $|z|<R_{n} /\left(\rho S^{n}\right)$ where $(1 / \rho)<R$. Finally, we see that (2.14) will hold under Hypothesis II when $0<|z|<R_{n} /\left(\rho S^{n}\right)$. Thus we have proved the following.

THEOREM 3.1. Assume Hypothesis II and let $f(z)$ be given by (2.1). Then $\left(\theta^{n} f\right)(z)$ is defined (by (2.3)) for $0<|z|<R_{n} /\left(\rho S^{n}\right)$ and is also given by (2.12), where $(1 / \rho)<R$.

THEOREM 3.2. Assume Hypothesis II and let $f(z)$ be given by (2.1) with $a_{-M} \neq 0$. Let $\tau$ and $\gamma$ be constants with $0<\tau<1$ and $0<\gamma<(1 / \rho)$. Then for all $n$ sufficiently large $\left(\theta^{n} f\right)(z)$ has no zero in $0<|z| \leq R_{n}\left(\tau^{n} / S^{n}\right) \gamma$.

Proof. In (2.14) put $z=R_{n}\left(\tau^{n} / S^{n}\right) x$, and write the result as

$$
\begin{aligned}
\left(\theta^{n} f\right)(z)= & A_{-M, n} z^{-M+n p}(2 \pi i)^{-1} \int_{|t|=\rho}(1 / t)^{M+1} f(1 / t) \\
& \times\left\{1+\sum_{j=0}^{\infty} \frac{A_{-M+P+j, n}}{A_{-M, n}}\left[\frac{R_{n} \tau^{n} x t}{S^{n}}\right]^{P+j}\right\} d t \\
= & A_{-M, n} z^{-M+n p} L_{n}(x),
\end{aligned}
$$

with $L_{n}(x)$ defined as indicated. This is valid for $0<|z|<R_{n} /\left(\rho S^{n}\right)$; i.e., $0<\tau^{n}|x t|=\tau^{n}|x| \rho<1$. In fact, take $0<|x| \leq \gamma<(1 / \rho)$, which implies $\tau|x| \rho<1$. For $x$ so restricted, and $0<\tau<1$, (3.2) implies that the sequence $\left\{L_{n}(x)\right\}_{n=1}^{\infty}$ converges uniformly to

$$
(2 \pi i)^{-1} \int_{|t|=\rho}(1 / t)^{M+1} f(1 / t) d t=a_{-M} \neq 0 .
$$

This produces a revised version of (2.17), namely

$$
\lim _{n \rightarrow \infty}\left\{\frac{z^{M-n p}\left(\theta^{n} f\right)(z)}{A_{-M, n} a_{-M}}\right\}=1, \quad 0<|z|=\left|R_{n} \tau^{n} x / S^{n}\right| \leq R_{n} \tau^{n} \gamma / S^{n},
$$

and the desired conclusion follows.

4. Examples. We calculate here some examples to illustrate the wide variety of situations covered by Theorems 2.2 and 3.2.

(i) $M=-1, p=0, \alpha_{k}=k, P=1, \theta=z d / d z$. We can use Hypothesis I since (2.9) reduces to

$$
\frac{\alpha_{2+j}}{\alpha_{1}}\left[\frac{\alpha_{1}}{\alpha_{2}}\right]^{1+j} \leq 1
$$


which is equivalent to $2+j \leq 2^{1+j}$, which holds for all $j \geq 0$. Now $R_{n}=A_{1, n} / A_{2, n}=(1 / 2)^{n}$, and therefore the discs $|z| \leq \gamma 2^{-n}$ are free of zeros of $\left(\theta^{n} f\right)(z)$ for $n$ large enough. For this operator, the change of variable $z=e^{w}, F(w)=f(z)=f\left(e^{w}\right)$ leads to $\left(\theta^{n} f\right)(z)=\left(D^{n} F\right)(w)$ where $D$ stands for ordinary differentiation. The half planes $\operatorname{Re}(w) \leq$ $\log \gamma-n \log 2$ are free of zeros of $D^{n} F$ for such $F$. This example was given in [13].

(ii) $M=-1, p=1, \alpha_{k}=k, P=1, \theta=z^{2} d / d z$. Hypothesis I applies again, for (2.9) becomes

$$
\frac{n+j+2}{n+1} \leq\left(\frac{n+2}{n+1}\right)^{j+1}
$$

but this is the same as

$$
1+\frac{j+1}{n+1} \leq\left(1+\frac{1}{n+1}\right)^{j+1}
$$

which is true by the binomial theorem. We calculate that $R_{n}=A_{1, n} / A_{2, n}$ $=n ! /(n+1) !=(n+1)^{-1}$, and so the discs $|z| \leq \gamma /(n+1)$ have no zeros of $\left(\theta^{n} f\right)(z)$. Putting $F(z)=f(1 / z)$, we obtain the zero-free regions for $F^{(n)}(z)$ recently described by R. P. Boas [2].

(iii) $M=1, p=0, \alpha_{k}=k, \theta=z d / d z$. Since $\alpha_{-1}=-1$ and $\alpha_{0}=0$ we have $P=2$. Condition (3.1) specializes to $1+j \leq S^{2+j}$, or $S \geq$ $(j+1)^{1 /(j+2)}$. The sequence $(j+1)^{1 /(j+2)}$ attains a maximum at $j=3$ and therefore Hypothesis II is satisfied for $S=4^{1 / 5}$. Since $R_{n}=1$, the regions $0<|z| \leq \gamma \tau^{n} / 4^{n / 5}$ are zero-free for $\theta$. This bound is asympotically best possible because of the example $f(z)=z^{-1}+z^{4}$, for which we have $\left(\theta^{n} f\right)(z)=(-1)^{n} z^{-1}+4^{n} z^{4}$.

(iv) $M=0, p=0, \alpha_{k}=2^{-k}, P=1$. Here the operator $\theta$ is given by $\left(\theta^{n} f\right)(z)=f\left(z / 2^{n}\right), n=0,1,2, \ldots$, which is defined for $|z|<2^{n} R$. The quantity on the left of $(2.9)$ is

$$
\frac{\alpha_{1+j}}{\alpha_{0}}\left[\frac{a_{0}}{\alpha_{1}}\right]^{1+j}=2^{-(1+j)}[2]^{1+J}=1,
$$

so that both sets of hypotheses are satisfied, the second for $S=1$. Since $R_{n}=2^{n}$, Theorem 2.2 says that the regions $0<|z| \leq \gamma 2^{n}(\gamma<R)$ are zero-free. Of course, this is equivalent to the fact that $f(z)$ itself has isolated zeros. This illustrates also the fact that the constant $\gamma$ depends on $f(z)$.

(v) $M=0, p=1, \alpha_{k}=1 /(k+1), P=1$. In this case $\theta$ is the anti-derivative operator, $\theta\left(z^{k}\right)=z^{k+1} /(k+1),(\theta f)(z)=\int_{0}^{z} f(s) d s$. Condition (3.1), dropping absolute values, becomes

$$
\frac{\alpha_{1+j+n}}{\alpha_{n}}\left[\frac{\alpha_{n}}{\alpha_{1+n}}\right]^{1+j} \leq S^{1+j}
$$


that is,

$$
\frac{n+1}{n+j+2}\left[\frac{n+2}{n+1}\right]^{j+1} \leq S^{j+1} .
$$

This inequality is false if $S=1$ (in fact the reverse inequality holds) which means that Hypothesis I fails. However Hypothesis II holds with $S=2$, which we get by setting $n=0$ and $j \rightarrow \infty$. Here $R_{n}=n+1$, giving zero-free regions of the form $|z| \leq(n+1) \gamma \tau^{n} / 2^{n}$.

(vi) $M=0, p \geq 0, \alpha_{k}=\Gamma(k+1) / \Gamma(k+p+1), P=1$. Applying $\theta$ termwise gives $(\theta f)(z)=\sum_{k=0}^{\infty} a_{k}[\Gamma(k+1) / \Gamma(k+p+1)] z^{k+p}$, and it follows that $\theta$ corresponds to the $p$ th order fractional integral. We use the definition of [8, p. 67]. If $p=1$ this example reduces to the previous one. The left side of (3.1) is asymptotically

$$
(n p+j)^{-p}(n p)^{p}\left[(n p)^{-p} S^{-1}(n p)^{p}\right]^{1+j}
$$

for large $n$ and $j$, where we have used the asymptotic formula $[\Gamma(n+a) / \Gamma(n+b)] \sim n^{a-b}([\mathbf{1}, \mathrm{p} .257])$. Consequently, Hypothesis II will hold for some $S \geq 1$, whose value we do not compute. After a calculation, $R_{n}=n p+1$. The zero-free regions for fractional integration are then $|z| \leq(n p+1) \gamma \tau^{n} / S^{n}$.

(vii) Let $G(w)$ be an entire function of finite exponential type $b$, so that $|G(w)|=O\left(e^{(b+\varepsilon)|w|}\right)$, for $\varepsilon>0$ and all large $w$. Fix the operator $\delta=z d / d z$ and consider, as a differential operator, the expression $G(\delta)$, which is defined by

$$
G(\delta) f=\lim _{n \rightarrow \infty} \sum_{j=0}^{n} \frac{G^{(j)}(0)}{j !} \delta^{j}(f) .
$$

This operator is considered by Hille [7, p. 48] in connection with questions on analytic continuation, and also by the first author [11]. One can show that, for $f(z)$ given by $(2.1)$,

$$
G(\delta) f(z)=\sum_{k=-M}^{\infty} a_{k} G(k) z^{k},
$$

with uniform convergences on compact subsets of $0<|z|<\mathrm{Re}^{-b}$. Thus $G(\delta)$ is a multiplier-sequence operator, $\theta=G(\delta)$, with $\alpha_{k}=G(k)$ and $p=0$. If we assume $G(-M) \neq 0$ and $G(-M+P) \neq 0$, then Hypothesis II will be satisfied for some $S$ because of the growth condition $G(k)=$ $O\left(e^{(b+\varepsilon) k}\right)$. Since $R_{n}=[G(-M) / G(-M+P)]^{n}$, then the zero-free regions for $\theta=G(\delta)$ are of the form $0<|z| \leq \gamma|\tau G(-M) / S G(-M+P)|^{n}$.

(viii) Now take $\theta=\delta=z d / d z$. The functions $P_{n}(z)=\delta^{n}\left(e^{z}\right) e^{-z}$ are known as Stirling polynomials ([11],[12]). By example (i) (the constant 
term disappears after one iteration, so we may as well take $M=-1$ ) the $n$th Stirling polynomial, for $n$ large enough, will have no zero inside $|z| \leq \gamma 2^{-n}$. The function $H(w)$ is the constant 1 , so that $I(x)=a_{1}=1$. Then (2.17) reduces to the asymptotic formula

$$
\lim _{n \rightarrow \infty} \frac{P_{n}\left(x / 2^{n}\right)}{\left(x / 2^{n}\right) \exp \left(x / 2^{n}\right)}=1, \quad|x| \leq \gamma<(1 / \rho) .
$$

It is shown in [11] that $\theta^{n}\left[\exp \left(z^{r}\right)\right]=P_{n}\left(z^{r}\right) \exp \left(z^{r}\right)$ and that the final set of $\exp \left(z^{r}\right)$ for $\theta$ (the set of all $\xi$ such that $\xi=\lim _{k \rightarrow \infty} \xi_{k}$ where $\theta^{n_{k}}\left[\exp \left(\xi_{k}^{r}\right)\right]=0$ for some sequence $\left.n_{k} \rightarrow \infty\right)$ consists of the rays from 0 going through the $r$ th roots of -1 . The fact, observed above, that $P_{n}\left(z^{r}\right) \neq$ 0 for $|z|<\left(\gamma / 2^{n}\right)^{1 / r}$ indicates the rate at which these rays are "filled in" with zeros of $P_{n}\left(z^{r}\right)$. Closely related results on the final set for derivatives of $\exp \left[-e^{z}\right]$ are found in [6].

5. Reciprocal powers. A completely dual theory to that of $\S 3$ exists for multiplier-sequence operators on power series

$$
f(z)=\sum_{k=-M}^{\infty} a_{k} z^{-k}=a_{-M} z^{M}+a_{-M+1} z^{M-1}+\cdots,|z|>T .
$$

We give a brief account of it in this section.

Let $p \geq 0$ and let $\beta$ be a function defined on all numbers of the form $-k+n p, k=-M,-M+1,-M+2, \ldots$ and $n=0,1,2, \ldots$, where $M$ is an integer. We continue to write $\beta_{-k+n p}=\beta(-k+n p)$. We assume that

$$
\begin{aligned}
& \beta_{-M} \neq 0, \text { and } \\
& \beta_{-k} \neq 0 \text { implies } \beta_{-k+n p} \neq 0, \quad n=0,1,2, \ldots,
\end{aligned}
$$

where $k=-M,-M+1, \ldots$. Assume that there is a first integer $P \geq 1$ such that

$$
\beta_{M-P} \neq 0 \text {. }
$$

We define the operator $\theta$ on the power basis by $\theta\left(z^{\nu}\right)=\beta_{\nu} z^{\nu+p}$, for $\nu$ in the domain of $\beta$, so that $\theta^{n}\left(z^{\nu}\right)=\beta_{\nu} \beta_{\nu+p} \cdots \beta_{\nu+(n-1) p} z^{\nu+n p}$. Letting $B_{\nu, n}=\beta_{\nu} \beta_{\nu+p} \cdots \beta_{\nu+(n-1) p}$ and applying $\theta^{n}$ termwise to (5.1), we arrive at the definition for $\theta^{n} f$, namely

$$
\left(\theta^{n} f\right)(z)=\sum_{k=-M}^{\infty} B_{-k, n} a_{k} z^{-k+n p}, \quad n=1,2,3, \ldots,
$$

whenever this series converges. Conditions (5.2), (5.3) (5.4) imply that

$$
\begin{aligned}
& B_{M, n} \neq 0, B_{M-P, n} \neq 0, \quad n=1,2,3, \ldots, \quad \text { and } \\
& B_{M-1, n}=\cdots=B_{M-P+1, n}=0, \quad \text { if } P>1 .
\end{aligned}
$$


We now state the condition corresponding to Hypothesis II. Hypothesis I has no practical correspondent since the standard operators $(\theta=$ $\left.z^{1+p} d / d z\right)$ involve negative $\beta_{\nu}$.

Hypothesis III. There exists a number $S>0$ such that

$$
\left|\frac{\beta_{M-P-\jmath+n p}}{\beta_{M+n p}}\right|\left|\frac{S \beta_{M+n p}}{\beta_{M-P+n p}}\right|^{P+j} \leq 1, \quad n, j=0,1,2, \ldots
$$

It follows that

$$
\left|\frac{B_{M-P-1, n}}{B_{M, n}}\right|\left|\frac{B_{M, n} S^{n}}{B_{M-P, n}}\right|^{P+j} \leq 1,
$$

and introducing $T_{n}=\left|B_{M-P, n} / B_{M, n}\right|$, (5.7) can be written

$$
\left|\frac{B_{M-P-\jmath, n}}{B_{M, n}}\right| \leq\left(T_{n} / S^{n}\right)^{P+j} ; \quad n=1,2,3, \ldots ; j=0,1,2, \ldots
$$

This condition implies that (5.5) converges and defines $\theta^{n} f$ for $|z|>$ $T T_{n} / S^{n}, n=1,2,3, \ldots$

The rest of the program parallels that of $\S 3$, and we give no further details except statements of pertinent theorems, which generalize corresponding results for differential operators in [14].

THEOREM 5.1. Assume Hypothesis III and let $f(z)$ be given by (5.1). Then $\left(\theta^{n} f\right)(z)$ is defined by (5.5) for $|z|>T_{n} /\left(\rho S^{n}\right)$ and is also given by

$$
\begin{aligned}
\left(\theta^{n} f\right)(z)= & B_{M, n} z^{M+n p}(2 \pi i)^{-1} \\
& \times \int_{|t|=\rho} t^{M-1} f(1 / t)\left\{1+\sum_{j=0}^{\infty} \frac{B_{M-P-\jmath, n}}{B_{M, n}}\left(\frac{1}{z t}\right)^{P+j}\right\} d t,
\end{aligned}
$$

where $(1 / \rho)>T$.

TheOrem 5.2. Assume Hypothesis III and let $f(z)$ be given by (5.1), with $a_{-M} \neq 0$. Let $\tau$ and $\gamma$ be constants with $0<\tau<1$ and $(1 / \gamma)>(1 / \rho)$ $>T$. Then for all $n$ sufficiently large, $\left(\theta^{n} f\right)(z)$ has no zero which satisfies $|z| \geq T_{n} /\left(S^{n} \tau^{n} \gamma\right)$.

EXAMPLE. If we take $\theta=G(\delta)$ as in example (vii), where $G(w)$ is entire and of finite exponential type $b$, and $f(z)$ a "balanced" Laurent series

$$
f(z)=\sum_{k=-M}^{M} C_{k} z^{k}, \quad M>0, \quad\left|C_{M}\right|=\left|C_{-M}\right|=1
$$


then the results both $\S 3$ and $\S 5$ apply. We assume $G(-M) G(M) \neq 0$ and, for simplicity, $G(-M+1) G(M-1) \neq 0$ so that $P=1$ for both sets of hypotheses. We have $\alpha_{-k}=\beta_{k}=G(k),-M \leq k \leq M$. From the growth conditions on $G(w)$ it is possible to choose a number $\mu>b$ so that (3.1) holds for an $S$ such that $|G(-M+1) S / G(-M)|=e^{\mu}$ and (5.6) holds for an $S^{\prime}$ such that $\left|G(M-1) / G(M) S^{\prime}\right|=e^{\mu}$. Since

$$
R_{n}=|G(-M) / G(-M+1)|^{n} \text { and } T_{n}=|G(M-1) / G(M)|^{n},
$$

the conclusions of Theorems 3.2 and 5.2 say that $\left(\theta^{n} f\right)(z)=(G(\delta))^{n} f(z)$ is zero-free when either

$$
0<|z| \leq \gamma\left(\tau / e^{\mu}\right)^{n} \quad \text { or }|z|^{-1} \leq \gamma\left(\tau / e^{\mu}\right)^{n} .
$$

These bounds become rather crude when one specializes $G$. Prather [11] has shown that if $G(w)=e^{i \lambda w} \prod_{k=1}^{\infty}\left(1-i w / w_{k}\right)$, where $\lambda$ is real and the $w_{k}$ are real and satisfy $\sum w_{w}^{-1}<\infty$, then the final set of a balanced $f(z)$ taken with respect to $\theta=G(\delta)$ lies on the unit circle. Consequently, the sets $0<|z| \leq r_{1}<1$, and $|z| \geq r_{2}>1$ are zero-free for all large $n$.

In two very interesting related papers, Craven and Csordas [4], [5] have results related to but not overlapping the results done here. In particular, they consider multiplier sequences $\left\{\gamma_{k}\right\}_{k=0}^{\infty}$ which do not increase the number of nonreal zeros of real polynomials. An example of multipliers which they take is $\gamma_{k}=Q(k)$, where $Q$ is a polynomial having real negative zeros, or more generallly an entire function of exponential type having real negative zeros of the form

$$
Q(z)=\exp (b z) \prod_{n=1}^{\infty}\left(1+z / a_{n}\right)
$$

say, where $b>0, \sum_{n=1}^{\infty}\left|a_{n}\right|^{-1}<\infty$. We allow the same multiplier in our example (vii) and the example after Theorem 5.2. When $M \leq 0$ in example (vii) so that $z=0$ is allowed and $\gamma_{k}=Q(k)$ as above, the asymptotic size of the zero free region shrinks to 0 , as $n \rightarrow \infty$. This is consistent with their results.

Moreover, if in example (vii), we take $G(w)$ to be an entire function of exponential type $b<\pi$, and $G$ vanishes at a set of integers from $\{-M$, $-M+1, \ldots\}$ having density 1 , yet not vanishing at all the integers (in light of Carlson's Theorem), then the boundary of convergence $|z|=$ $R \exp (-b)$ of $G(\delta) f(z)$, given in (4.2), is a natural boundary, by the Fabry gap theorem (see e.g. Bieberbach [3], Satz 2.21 or Hille [7], Theorem 11.7.2). Our hypotheses $(G(-M) \neq 0, G(-M+P) \neq 0)$ do not allow $G$ to vanish at all the integers. Consequently, the boundary $|z|=R \exp (-b m)$ of the domain of convergence for $(G(\delta))^{m} f(z)$ is a natural boundary, for all $m=2,3, \ldots$. 


\section{REFERENCES}

1. M. Abramowitz and I. Stegun (Eds.), Handbook of Mathematical Functions with Formulas, Graphs and Mathematical Tables, Nat. Bureau Standards Appl. Math. Ser., No. 55, Superintendent of Documents, U.S. Gov. Printing Office, Washington, D.C., 1964. MR 29 \#4914.

2. R. P. Boas, Zeros of Successive Derivatives of a Function Analytic at Infinity, Univ. Beograd. Publ. Electrotehn. Fak., Ser. Mat. Fiz. No. 606, (1978), 51-52.

3. L. Bieberbach, Analytische Fortsetzung, Ergebnisse der Mathematik, New Series, Nos. 3, Springer-Verlag, Berlin, 1955.

4. T. Craven and G. Csordas, An inequality for the distribution of zeros of polynomials and entire functions, Pacific J. Math., 95 (2) (1981), 263-280.

$5 . \quad$ Zero-diminishing linear transformations, Proc. Amer. Math. Soc., 80 (1980), $544-546$.

6. A. Edrei, Zeros of successive derivatives of entire functions of the form $H(z) e^{-e^{z}}$, Trans. Amer. Math. Soc., (1980), 207-226.

7. E. Hille, Analytic Function Theory, Vol. II, Ginn, Boston, 1962.

8. K. Oldham and J. Spanier, The Fractional Calculus, Academic Press, New York, 1974.

9. E. L. Post, Generalized differentiation, Trans. Amer. Math. Soc., 32 (1930), 723-781.

10. C. L. Prather, On the zeros of derivatives of balanced trigonometric polynomials, Pacific J. Math., 81 (2) (1979), 512-522.

11. __ On some new and old theorems on final sets, Houston J. Math., 7 (1981), 407-430.

12. L. A. Rubel and B. A. Taylor, A completeness theorem for entire functions, J. Indian Math. Soc., 23 (3-4) (1967), 195-198.

13. J. K. Shaw and C. L. Prather, Zeros of successive derivatives and iterated operators on analytic functions, Proc. Amer. Math. Soc., 79 (1980), 225-232.

14. Z_ Zeros of successive derivatives of functions analytic in a neighborhood of a single pole, Michigan Math. J., 29 (1982), 111-119.

15. D. V. Widder, The inversion of the Laplace integral and the related moment problem, Trans. Amer. Math. Soc., 36 (1934), 107-200.

16. _ The Laplace Transform, Princeton University Press, Princeton, N.J., 1941.

17. The Convolution Transform, Princeton Unviersity Press, Princeton, N.J., 1955.

18. __ An Introduction to Transform Theory, Academic Press, New York, 1971.

Received June 2, 1980.

VIRGINIA TECH

BLACKSBURG, VA 24061-4097 


\section{PACIFIC JOURNAL OF MATHEMATICS \\ EDITORS}

DONALD BABBITT (Managing Editor)

University of California

Los Angeles, CA 90024

\section{Hugo Rossi}

University of Utah

Salt Lake City, UT 84112

C. C. Moore and Arthur Ogus

University of California

Berkeley, CA 94720
J. DugundiI

Department of Mathematics

University of Southern California

Los Angeles, CA 90089-1113

R. FINN and H. SAMELSON

Stanford University

Stanford, CA 94305

ASSOCIATE EDITORS
R. ARens
E. F. BECKENBACH
B. H. NeumanN
F. WOLF
K. YoshidA (1906-1982)

\section{SUPPORTING INSTITUTIONS}

UNIVERSITY OF ARIZONA

UNIVERSITY OF BRITISH COLUMBIA

CALIFORNIA INSTITUTE OF TECHNOLOGY

UNIVERSITY OF CALIFORNIA

MONTANA STATE UNIVERSITY

UNIVERSITY OF NEVADA, RENO

NEW MEXICO STATE UNIVERSITY

OREGON STATE UNIVERSITY
UNIVERSITY OF OREGON

UNIVERSITY OF SOUTHERN CALIFORNIA

STANFORD UNIVERSITY

UNIVERSITY OF HAWAII

UNIVERSITY OF TOKYO

UNIVERSITY OF UTAH

WASHINGTON STATE UNIVERSITY

UNIVERSITY OF WASHINGTON 


\section{Pacific Journal of Mathematics}

Vol. 104, No. 1

May, 1983

Nestor Edgardo Aguilera and Eleonor Ofelia Harboure de Aguilera, On

the search for weighted norm inequalities for the Fourier transform $\ldots \ldots .1$

Jin Akiyama, Frank Harary and Phillip Arthur Ostrand, A graph and its complement with specified properties. VI. Chromatic and achromatic numbers ......................................... 15

Bing Ren Li, The perturbation theory for linear operators of discrete type . . . 29

Peter Botta, Stephen J. Pierce and William E. Watkins, Linear

transformations that preserve the nilpotent matrices .............. 39

Frederick Ronald Cohen, Ralph Cohen, Nicholas J. Kuhn and Joseph

Alvin Neisendorfer, Bundles over configuration spaces .......... 47

Luther Bush Fuller, Trees and proto-metrizable spaces . . . . . . . . . . 55

Giovanni P. Galdi and Salvatore Rionero, On the best conditions on the

gradient of pressure for uniqueness of viscous flows in the whole space . . 77

John R. Graef, Limit circle type results for sublinear equations $\ldots \ldots \ldots \ldots 85$

Andrzej Granas, Ronald Bernard Guenther and John Walter Lee,

Topological transversality. II. Applications to the Neumann problem for

$y^{\prime \prime}=f\left(t, y, y^{\prime}\right) \ldots \ldots \ldots \ldots \ldots \ldots \ldots \ldots \ldots \ldots \ldots \ldots \ldots \ldots \ldots . \ldots 5$

Richard Howard Hudson and Kenneth S. Williams, Extensions of

theorems of Cunningham-Aigner and Hasse-Evans . . . . . . . . . . . 111

John Francis Kurtzke, Jr., Centralizers of irregular elements in reductive algebraic groups

James F. Lawrence, Lopsided sets and orthant-intersection by convex

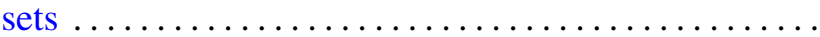

Åsvald Lima, G. H. Olsen and U. Uttersrud, Intersections of $M$-ideals and

$G$-spaces

Wallace Smith Martindale, III and C. Robert Miers, On the iterates of derivations of prime rings

Thomas H. Pate, Jr, A characterization of a Neuberger type iteration procedure that leads to solutions of classical boundary value problems

Carl L. Prather and Ken Shaw, Zeros of successive iterates of multiplier-sequence operators

Billy E. Rhoades, The fine spectra for weighted mean operators

Rudolf J. Taschner, A general version of van der Corput's difference theorem

Johannes A. Van Casteren, Operators similar to unitary or selfadjoint ones 